\title{
Myelin vs Axon Abnormalities in White Matter in Bipolar Disorder
}

\author{
Kathryn E Lewandowski*,1,2, Dost Ongür ${ }^{1,2}$, Sarah H Sperry', Bruce M Cohen ${ }^{1,2}$, Selma Sehovic', \\ Jacqueline R Goldbach' and Fei Du' ${ }^{1,2}$ \\ 'McLean Hospital Schizophrenia and Bipolar Disorder Program, Belmont, MA, USA; ${ }^{2}$ McLean Hospital, Harvard Medical School, Boston, \\ MA, USA
}

\begin{abstract}
White matter (WM) abnormalities are among the most commonly reported neuroimaging findings in bipolar disorder. Nonetheless, the specific nature and pathophysiology of these abnormalities remain unclear. Use of a combination of magnetization transfer ratio (MTR) and diffusion tensor spectroscopy (DTS) permits examination of myelin and axon abnormalities separately. We aimed to examine myelination and axon geometry in euthymic patients with bipolar disorder with psychosis (BDP) by combining these two complementary noninvasive MRI techniques. We applied a combined MRI approach using MTR to study myelin content and DTS to study metabolite (Nacetylaspartate, NAA) diffusion within axons in patients with $\operatorname{BDP}(n=21)$ and healthy controls $(n=24)$. Data were collected from a $1 \times 3 \times 3-\mathrm{cm}$ voxel within the right prefrontal cortex WM at 4 Tesla. Clinical and cognitive data were examined in association with MTR and DTS data. MTR was significantly reduced in BDP, suggesting reduced myelin content. The apparent diffusion coefficient of NAA did not differ from healthy controls, suggesting no changes in axon geometry in patients with BDP. These findings suggest that patients with BDP exhibit reduced myelin content, but no changes in axon geometry compared with controls. These findings are in contrast with our recent findings, using the same techniques, in patients with schizophrenia (SZ), which suggest both myelination and axon abnormalities in SZ. This difference may indicate that alterations in WM in BDP may have unique causes and may be less extensive than WM abnormalities seen in SZ.

Neuropsychopharmacology (2015) 40, 1243-1249; doi:I0.1038/npp.2014.310; published online 17 December 2014
\end{abstract}

\section{INTRODUCTION}

White matter (WM) abnormalities are among the most consistent neuroimaging findings in bipolar disorder (BD; Vederine et al, 2011) and include WM hyperintensities (Kempton et al, 2008; Beyer et al, 2009) decreased density, (Bruno et al, 2004; McDonald et al, 2004; McIntosh et al, 2005; Stanfield et al, 2009), and, more recently, reduced integrity of WM tracts (Sarrazin et al, 2014). Diffusion tensor imaging (DTI) techniques have provided evidence for WM abnormalities especially in frontal regions (for example, (Adler et al, 2004; Beyer et al, 2005; McIntosh et al, 2005; Yurgelun-Todd et al, 2007; Linke et al, 2013)) but also global WM deficits and abnormalities in longitudinal tracts (McIntosh et al, 2008; Emsell et al, 2013; Torgerson et al, 2013). A recent metaanalysis of whole-brain DTI studies reported widespread WM abnormalities with decreased fractional anisotropy (FA) in patients with BD (Nortje et al, 2013). WM integrity is associated with behavioral outcomes in $\mathrm{BD}$, including

\footnotetext{
*Correspondence: Dr KE Lewandowski, McLean Hospital, Harvard Medical School, AB 328, I 5 Mill Street, Belmont, MA 02492, USA, Tel: + 617855 2886, Fax: + 6178552895 ,

E-mail: klewandowski@mclean.harvard.edu

Received 7 August 2014; revised 22 October 2014; accepted II November 2014; accepted article preview online 20 November 2014
}

cognitive functioning. DTI parameters are associated with poorer executive functioning in patients with $\mathrm{BD}$ and their relatives (Linke et al, 2013; Oertel-Knochel et al, 2014).

The specific nature and pathophysiology of these abnormalities remain unclear. DTI measures, which are commonly interpreted as indices of 'white matter integrity,' are relatively nonspecific, and do not clearly differentiate between axon- and myelin-specific abnormalities (Mori and Zhang, 2006). This is because DTI quantifies diffusion of water molecules and water exists in both intracellular and extracellular spaces (with exchange between the two). We recently employed a novel technique for differentiating these abnormalities using a combination of magnetization transfer ratio (MTR) and diffusion tensor spectroscopy (DTS; Du et al, 2013). MTR measures magnetization exchange of protons between 'free' water molecules and water molecules that are 'bound' to myelin lipids. The more myelin present, the more proton exchange occurs, and the higher the MTR. MTR measures proton exchange between free water and water bound to any macromolecule moieties and is therefore not entirely specific to the myelin. However, as myelin constitutes the overwhelming majority of macromolecules in the brain's WM (Laule et al, 2007), MTR in the WM is considered an acceptable metric of myelin content. DTS quantifies the diffusion signal from $\mathrm{N}$-acetylaspartate (NAA) and other metabolites. In addition, NAA is located 
exclusively in neurons and almost exclusively in the cytosol of these cells (whereas other measurable metabolites such as Creatine and Choline are associated with glia, as well as neurons, and with enzymes and cell membranes in all cell types), and thus the NAA signal provides specific information about intraneuronal space. This combination of techniques permits the examination of both axon- and myelin-related WM abnormalities, separately, in vivo. In our combined MTR-DTS study, we found significantly reduced MTR (suggesting reduced myelin content) and significantly elevated apparent diffusion coefficient (ADC) of NAA (suggesting abnormal intraneuronal geometry) in schizophrenia (SZ) patients compared with age- and sex-matched healthy control participants.

BD patients who experience psychotic symptoms (BDP) generally have a more severe phenotype of $\mathrm{BD}$ and manifest the same WM abnormalities described above for $\mathrm{BD}$ in general. In fact, BDP patients also share some WM abnormalities with SZ patients based on DTI (Sussmann et al, 2009). A comparison of BDP and SZ patients found WM abnormalities in both groups, which did not differ from each other (Cui et al, 2011). However, it is not known whether or not patients with BDP exhibit both the myelin and axon abnormalities seen in SZ.

In this study, we examined myelination and axon geometry in euthymic patients with BDP by combining two complementary noninvasive MRI techniques-MTR and DTS - in BDP and healthy controls. Specifically, based on findings of myelination abnormalities in BDP (Tkachev et al, 2003; Regenold et al, 2007; Sokolov, 2007) we hypothesized that the myelin component, as estimated by MTR, would be reduced in BDP. As this is the first study to explicitly probe axon geometry in BDP using DTS, we did not make specific predictions regarding NAA ADC changes.

\section{MATERIALS AND METHODS}

\section{Participants}

Participants included 21 patients with BD I with psychosis (BDP) and 24 age- and sex-matched healthy control participants. Patients were recruited through the Psychotic Disorders Division at McLean Hospital; controls were recruited from the community via fliers and advertisements. All procedures were approved by the McLean Hospital IRB. Patients with BDP were stable outpatients at the time of assessment, defined as having been out of the hospital for at least 1 month before enrollment with Positive and Negative
Syndrome Scale (PANSS) scores $<75$, ratings of 3 (mild) or less on the PANSS psychosis items, and Young Mania Rating Scale (YMRS) scores below 6. Patients with rapid cycling BD were not eligible. All patients were taking psychiatric medications at the time of testing. To reduce medication confounds, only patients on monotherapy lithium or valproate plus an atypical antipsychotic (excluding clozapine) were eligible. Diagnosis was determined by a trained clinician using the SCID-IV diagnostic interview in conjunction with all available collateral information from medical records, treatment providers, and family members.

\section{Procedures}

Cognitive and clinical assessment. Cognitive functioning was assessed using the MATRICS Consensus Cognitive Battery (MCCB; Nuechterlein et al, 2008). While the МССВ was developed for cognitive assessment in SZ, recent work has demonstrated its sensitivity and validity in detecting neurocognitive deficits in patients with BDP (Burdick et al, 2011). The MCCB includes 10 tasks that measure processing speed (Brief Assessment of Cognition in Schizophrenia Symbol Coding, Animal Fluency, Trails A), attention (Continuous Performance Test), working memory (WMSIII Spatial Span, Letter-Number Span), verbal learning (Hopkins Verbal Learning Test-Revised), visual learning (Brief Visuospatial Memory Test-Revised), problem-solving (Neuropsychological Assessment Battery), and social cognition (Mayer-Salovey-Caruso Emotional Intelligence Test). Total administration time is $60-90 \mathrm{~min}$. The North American Adult Reading Test (NAART) was administered as an estimate of premorbid intelligence. All neurocognitive scores were converted to standardized scores based on age-adjusted norms. Clinical and community functioning assessment included the following: the PANSS; YMRS; MontgomeryAsberg Depression Rating Scale (MADRS); Multnomah Community Ability Scale (MCAS); and the Social and Occupational Functioning Assessment Scale (SOFAS). The MCAS measures functioning in multiple domains (for example, social interest/effectiveness, independence, and meaningful activities). The SOFAS is a 100-point scale similar to the GAF that evaluates social and occupational functioning not directly influenced by psychological symptoms.

MTR. Data were collected from a $1 \times 3 \times 3-\mathrm{cm}$ voxel within the right prefrontal cortex WM (Figure 1) at 4 Tesla (Varian/UnityInova, Varian, Palo Alto, CA, USA). We chose

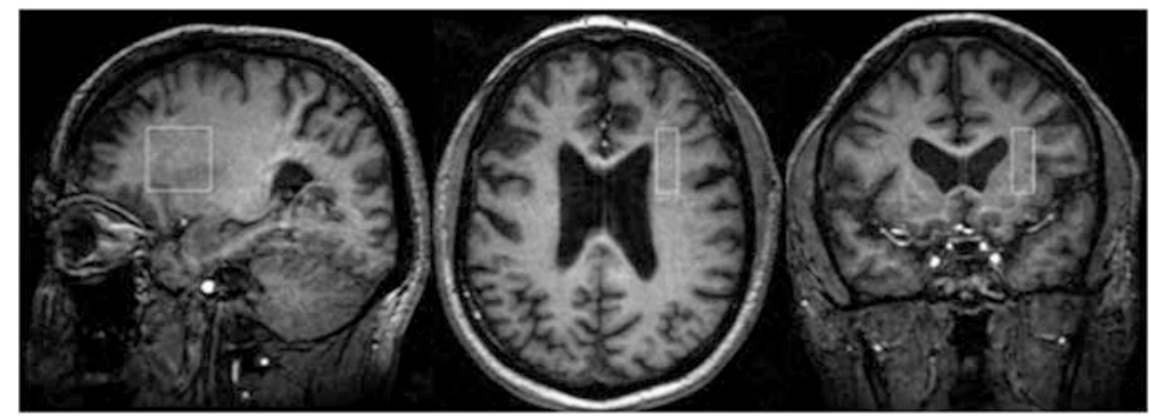

Figure I Representative images depicting the location of our $1 \times 3 \times 3$ - white matter voxel in the right prefrontal cortex of a patient. 
a single voxel approach to measure MTR because we aimed to examine MTR and DTS data from the same voxel. Briefly, the voxel was placed in the corona radiate, centered at the level of and lateral to the genu of the corpus callosum so as to exclude callosal fibers. The mediolateral extent was $1 \mathrm{~cm}$; anteroposterior and dorsoventral extents were $3 \mathrm{~cm}$. The voxel was positioned in pure WM, anchored by adjacent gray matter in anterior and lateral directions to ensure comparable locations across scans. The DTS signal we utilize in this study arises from NAA. This metabolite is present in the brain in a concentration that is $\sim 5000$-fold lower than that of water. Therefore, although the NAA signal is reliable, in order to obtain accurate data for our purposes we need to integrate data over time (leading to long scan times) and space (leading to larger voxels).

A test-retest study of 10 healthy subjects was conducted and within-subject coefficients of variation indicated excellent agreement between the two scans $(7.0 \%$ for MTR, 3.4\% for NAA ADC, and 1.5\% for water ADC). For details of anatomical imaging, choice of voxel placement, and the test-retest study see ( $\mathrm{Du}$ et al, 2013). We used a BISTRO saturation pulse train (deGraff et al, 1996) constructed with multiple hyperbolic Sec pulses (width $50 \mathrm{~ms}$ ) with varied radiofrequency pulse amplitudes and applied at the beginning of a standard point-resolved spectroscopy (PRESS) sequence (before the 90-degree pulse) to saturate 'bound-water' signal with a specific frequency offset (Chen et al, 1995; deGraff et al, 1996). Data were obtained in $50-\mathrm{Hz}$ steps at a range of frequencies offset $400-1000 \mathrm{~Hz}$ in either direction from the water signal, and a single MTR number was calculated by averaging across frequencies. Saturation time $\left(t_{\text {sat }}\right)$ was $2.6 \mathrm{~s}$ with repetition time/echo time of $3000 / 30 \mathrm{~ms}$ and two repetitions.

DTS. To enhance comparisons with previous studies and assure validity of measures, the procedures followed those of Du et al (2013). Briefly, the standard PRESS sequence was modified by incorporating diffusion gradients for DTS measurements (Figure 2). Bipolar diffusion gradients with six directions and one control (totaling seven spectra) were applied to calculate diffusion tensors of signal from water and metabolites. The applied $b$ value was $1412 \mathrm{~s} / \mathrm{mm}^{2}$, calibrated by a phantom with water ADC assumed to be $2.1 \times 10^{-3} \mathrm{~mm}^{2} / \mathrm{s}$ at room temperature (Kan et al, 2012). Repetition time/echo time was $3000 / 135 \mathrm{~ms}$, and diffusion time $\left(D_{\mathrm{t}}\right)$ was $60 \mathrm{~ms}$. There were 96 repetitions for metabolites and 4 repetitions for water measurements. Metabolite spectra were acquired with water saturation with VAPOR (Tkac et al, 1999). In order to correct frequency and phase draft as well as eddy current effect resulting from hardware variations, subject movement or diffusion gradients, each scan (individual FID, free induction decay) was digitized before summing all FIDs.

Total experiment time for MTR plus DTS measurements was $\sim 70 \mathrm{~min}$. Water data from the DTS experiment were comparable to the literature, although from a larger voxel. Between human subject studies we carried out a phantom DTS to calibrate the $b$ value.

MRI and MRS data processing/analysis. All data-processing techniques were conducted using our previously

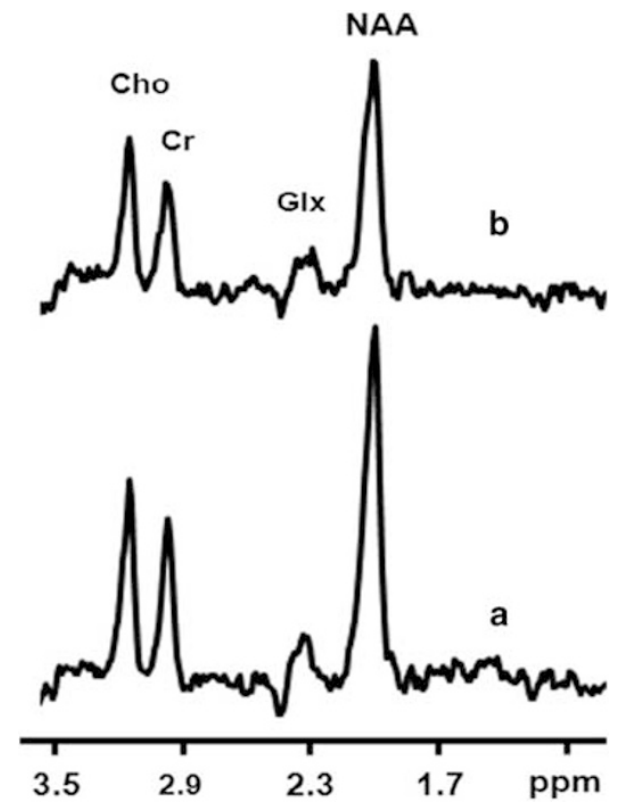

Figure 2 Sample spectra: one representative subject ' $H$ spectra acquired from one representative subject. (a) Control without diffusion gradients. (b) Diffusion weighted (diffusion gradients were placed along $x$ and $y$ directions). Cho $=$ Choline; $C R=$ Creatinine; $G l x=$ glutamate and glutamine; $\mathrm{NAA}=\mathrm{N}$-acetylaspartate.

reported methods (Du et al, 2013). Briefly, all MRI/MRS data were processed by an MR physicist (FD) who was blind to diagnosis. Post-processing of the FIDs including apodization, Fourier transformation, frequency, and phase correction as well as calculation of MTR and DTS constants was carried out using the software available in the Varian Console and home-grown software running on MATLAB. As MTR and DTS measurement both involve relative signal change, we digitized the water or NAA signal and normalized it to baseline. Reported units for ADC, RD, and $\mathrm{AD}$ are in $\mathrm{mm}^{2} / \mathrm{s} \times 10^{-3}$.

\section{Statistical Approach}

All analyses were performed using STATA (V. 12) and SPSS (V. 18). First, we conducted two-sample $t$-tests and $\chi^{2}$ tests to compare control and bipolar samples on demographic characteristics. To test our primary hypotheses of (a) reduction in MTR and (b) abnormalities of NAA ADC in $\mathrm{BDP}$, we ran a series of $t$-tests comparing groups on these measures. We also carried out secondary analyses of NAA $\mathrm{RD}, \mathrm{AD}$, and $\mathrm{FA}$, and water $\mathrm{ADC}, \mathrm{RD}, \mathrm{AD}$, and FA. Lastly, we performed post hoc analyses to examine possible effects of age, sex, BMI, and education on associations between WM measures and diagnostic group, as all of these might be associated with differences in WM.

We performed a correlational analysis examining associations among diffusion variables in order to detect possible structured covariance. We also examined associations between MTR and NAA ADC with demographic variables (age; sex; and education), clinical variables (duration of illness; number of lifetime hospitalizations; PANSS; YMRS; MADRS; and CPZ equivalents), community functioning (MCAS andSOFAS), and cognitive functioning (MCCB 
scores). We chose not to correct these correlation analyses for multiple comparisons in this exploratory study in order to detect even modest relationships, which may be pursued as to replicability in future work.

\section{RESULTS}

Demographic and clinical data are presented in Table 1. Groups did not differ on age and sex. Patients with BDP had significantly lower educational attainment and higher BMI than controls.

\section{MTR and DTS}

MTR and DTS results are presented in Table 2. We found a significant reduction of MTR in BDP compared with healthy controls $(t(40)=-2.65 ; p=0.006)$, which remained significant after controlling for age $(\mathrm{F}(1,38)=3.86, p=0.03)$. There was no significant difference between patients with $\mathrm{BDP}$ and controls in NAA ADC, which remained unchanged after controlling for age. In secondary analyses, groups did not differ on NAA RD, NAA AD, or NAA FA (see Table 2). However, groups did differ significantly on measures of Water RD (BD > Control; $t(42)=3.95 ; p=0.0003$ ), Water $\mathrm{ADC}(\mathrm{BD}>\mathrm{Control} ; t(42)=3.58 ; p=0.0009)$, and Water FA $(\mathrm{BD}<$ Control; $t(42)=-2.43 ; p=0.02)$.

MTR was not correlated with NAA ADC or any other NAA measures; similarly, MTR was not correlated with any water diffusion measure, although there was a trend-level association between MTR and Water RD $(r=-0.29$, $p=0.07)$. NAA ADC was correlated with Water $\mathrm{RD}$ $(r=0.46, p=0.002)$, Water ADC $(r=0.38, p=0.01)$ and negatively with Water FA $(r=-0.39, p=0.01)$.

Post hoc ANCOVAS were performed to examine the potential effects of BMI and education, which differed by group. Sex was also examined as a potential confounder as the groups were not matched for this variable (although differences were nonsignificant). BMI and sex were not associated with any of our WM outcomes, and group differences remained after accounting for these variables. Including education as a covariate did not affect associations between NAA or Water measures and group; however, after accounting for education, group differences in MTR no longer reached statistical significance $(F(1,38)=2.22$, $p=0.15)$.

\section{MTR, DTS, and Demographic, Clinical, and Cognitive Variables}

In the total sample, NAA ADC was correlated with age $(r=0.33, p=0.03)$. MTR was associated with educational attainment $(r=0.44, p=0.004)$. We examined correlations among MTR, DTS, and clinical, cognitive, and community functioning variables in the BDP sample only. In terms of clinical variables, NAA ADC was correlated with age of onset $(r=0.48, p=0.05)$ and negatively correlated with PANSS Positive scores $(r=-0.45, p=0.04)$ and PANSS total score $(r=-0.50, p=0.04)$; NAA ADC showed a trendlevel association with PANSS general scores $(r=-0.45$, $p=0.06$ ) but was not associated with any other clinical measures. MTR was associated with YMRS $(r=-0.46$, $p=0.04)$. Neither MTR nor NAA ADC was associated with $\mathrm{CPZ}$ equivalents or community-functioning measures.

Neither MTR nor NAA ADC were significantly correlated with the NAART score or any MCCB cognitive domain, although there was a trend level association between Working Memory and MTR $(r=0.37 ; p=0.10)$.

We conducted a set of post hoc analyses to examine potential associations between Lithium and our WM measures. Of our subjects, 14 of 21 were prescribed Lithium (based on self-report). Group-wise comparisons of MTR and NAA ADC in patients on $v s$ off of Lithium showed no differences in MTR $(t=0.69 ; p=0.50)$; however, we did find a significant difference in NAA ADC, with patients prescribed Lithium showing lower mean values $(t=2.80 ; p=0.01)$.

Table I Demographic and Clinical Characteristics of Study Participants

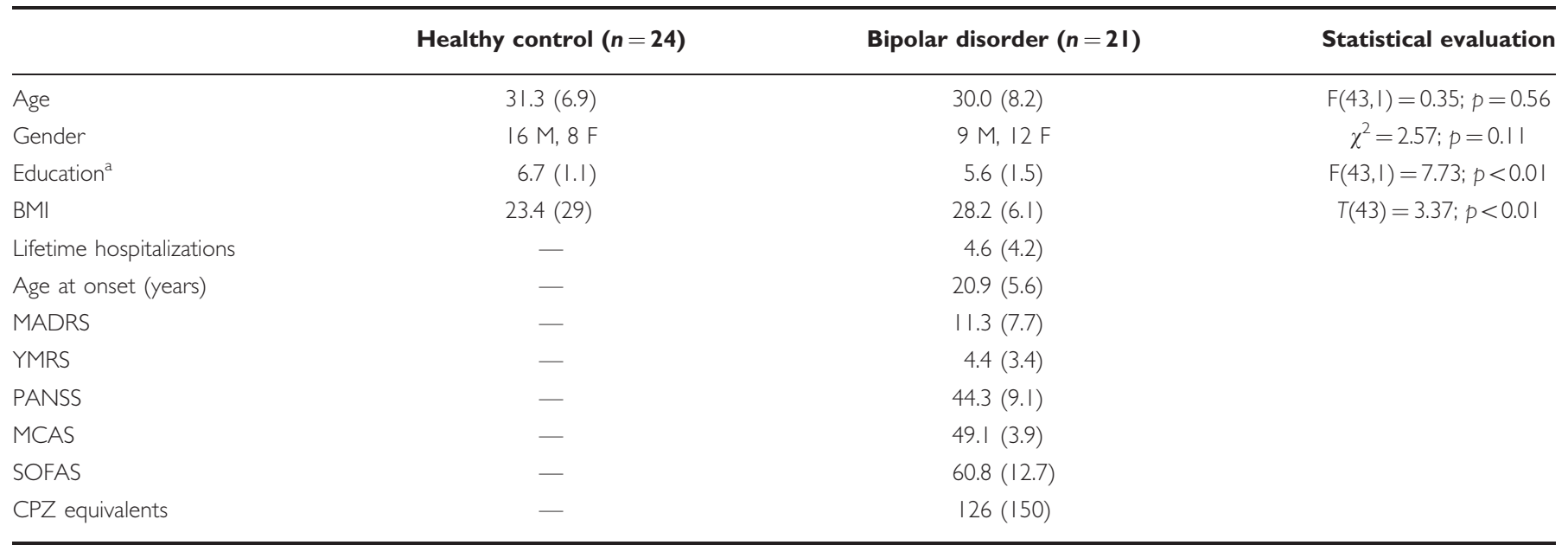

Abbreviations: CPZ, chlorpromazine; MADRS, Montgomery-Åsberg Depression Rating Scale; MCAS, Multnomah Community Ability Scale; PANSS, Positive and Negative Syndrome Scale; SOFAS, Social and Occupational Functioning Assessment Scale; YMRS, Young Mania Rating Scale.

aEducation code: 3: graduated high school; 4: part college; 5: graduated 2-year college; 6: graduated 4-year college; 7: part graduate/professional school; 8: completed graduate/professional school. 
Table 2 MTR and DTS Data Summary

\begin{tabular}{|c|c|c|c|c|}
\hline & Healthy control $(n=24)$ & Bipolar disorder $(n=2 I)$ & Test statistic $^{a}$ & Effect size (Cohen's d) \\
\hline MTR & $0.17(0.02)$ & $0.15(0.02)$ & $t(40)=-2.65 ; p=0.006 * *$ & 1.05 \\
\hline NAA RD & $0.15(0.04)$ & $0.18(0.07)$ & $t(40)=1.49 ; p=0.143$ & 0.50 \\
\hline NAA AD & $0.34(0.09)$ & $0.36(0.11)$ & $t(40)=0.70 ; p=0.486$ & 0.20 \\
\hline NAA ADC & $0.21(0.05)$ & $0.24(0.07)$ & $t(40)=1.49 ; p=0.143$ & 0.50 \\
\hline NAA FA & $0.48(0.16)$ & $0.43(0.18)$ & $t(40)=-0.94 ; p=0.355$ & 0.30 \\
\hline Water ADC & $0.61(0.05)$ & $0.66(0.04)$ & $t(42)=3.58 ; p=0.0009 * * *$ & 1.00 \\
\hline Water FA & $0.23(0.11)$ & $0.16(0.07)$ & $t(42)=-2.43 ; p=0.02 *$ & 0.70 \\
\hline
\end{tabular}

* $p<0.05$; *** $p<0.01$; ***** $p<0.001$.

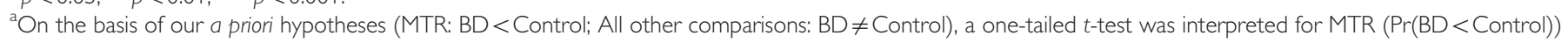
and two-tailed tests were reported for all other comparisons $(\operatorname{Pr}(|\mathrm{BD}| \neq \mid$ Control $\mid))$.
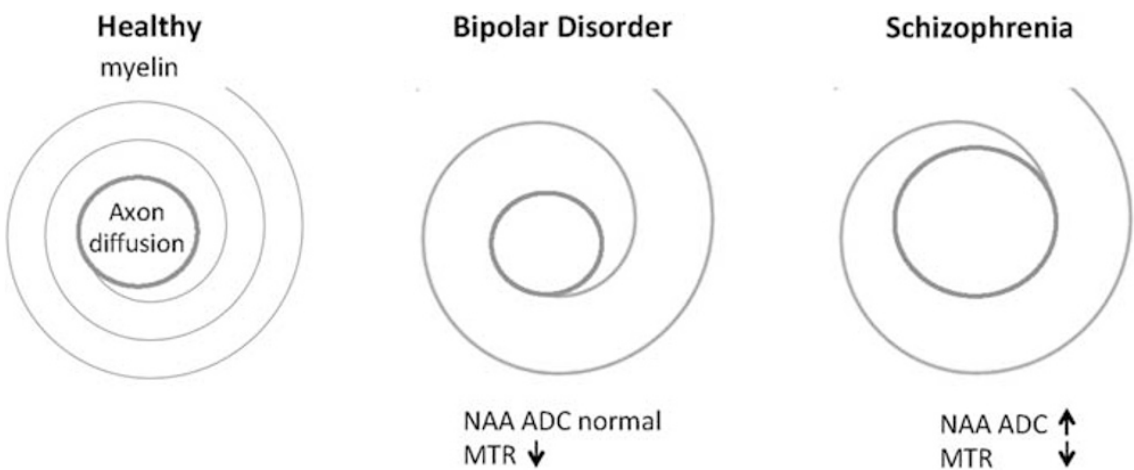

Figure 3 Comparison of schizophrenia (SZ) and bipolar disorder (BD) findings based on N-acetylaspartate (NAA) and magnetization transfer ratio (MTR) measures.

\section{DISCUSSION}

In this study, we examined WM integrity in BDP using two complementary MRI-based techniques: MTR to probe myelin content and DTS to probe axonal geometry. Our findings suggest that there is a reduction in myelin content in BDP but that axonal geometry is not significantly abnormal. This pattern is distinct from what we reported in SZ, where both WM measures showed abnormalities (Du et al, 2013). While DTI studies have revealed WM abnormalities in both SZ and BDP, the present MTR/DTS findings suggest that there may be distinctions in the underlying biology of WM pathology in the two conditions.

Finding abnormalities in myelin but not axons suggests that WM in BDP may be characterized by dynamic abnormalities. The synthesis and maintenance of myelin is an active process throughout life and it is supported by neuronal activity (Liu et al, 2012; Makinodan et al, 2012). This process appears to be abnormal in BDP. Changes in axonal geometry, by contrast, would reflect structural problems in the maintenance of axon biology and perhaps more permanent abnormalities in neuronal function. We did not find evidence for such changes in BDP. Our previous findings in SZ suggested that abnormalities are present not only in the amount of myelin but also in the structure of the long axons of neurons, whereas our current work indicates that WM abnormalities in BDP may be specific to myelin. Myelin has a continuing turnover, and it is possible that the abnormalities in $\mathrm{BD}$, sparing the long axons of neurons, could be more dynamic and potentially mutable (Figure 3). It is intriguing that this difference is similar to the course of the two illnesses, specifically that $\mathrm{BDP}$ is a recurrent disorder with interepisode recovery, whereas SZ is typified by chronic symptoms.

A reduction in MTR accompanied by a normal NAA ADC provides a mismatch between axonal geometry and the myelin component. This would lead to abnormal signal transduction in BDP, even though axon geometry may be normal. We recently discussed the implications of myelin/ axon interactions in this process (Du and Ongur, 2013). Although our findings suggest that signal transduction may be abnormal in BDP, they do not provide information on the precise nature of this abnormality. Nonetheless, we can say that the pathology appears distinct from that seen in SZ where both myelin and axon abnormalities were seen.

The correlations we report between NAA ADC and demographic and clinical variables are intriguing. However, NAA ADC was not significantly abnormal in the BDP group. 
In addition, these statistical findings would not be significant if corrected for multiple comparisons. It is necessary to explore these relationships in a larger patient sample before drawing firm conclusions from them. It is also notable that we did not observe any relationships between either MR measure and cognitive measures. Such relationships have been reported in DTI studies. Our negative finding may be due to a lack of power, or the fact that we examined a single WM region that may not be critical for the cognitive measures utilized. Our finding that education was associated with MTR but not NAA ADC and that this relationship may moderate the group findings with regard to MTR warrants further investigation and may speak to the dynamic nature of myelination and its potential associations with cognitive and functional outcomes.

This study has several other limitations. The major one for interpretation of our findings is that our BDP patients were taking medication. We have no ability to definitively rule out medication effects on our measures, which could be driving or masking associations between WM signals, diagnosis, and other measures. Nonetheless, some evidence suggests that medication effects are not pronounced in our study, and that some potential medication effects may actually be in the direction of normalization. In our sample, post hoc analyses of patients on $v s$ off of Lithium showed no differences in MTR between groups; however, patients prescribed Lithium had significantly lower mean NAA ADC values. Thus, our MTR findings do not appear to be driven by Lithium use. Whereas NAA ADC did not differ from controls, there are some suggestions that within patients with BDP Lithium use may be associated with normalization of axon diameter. These findings are preliminary and warrant further investigation. In addition, there was no relationship between chlorpromazine equivalents and our MRI/MRS measures in this patient sample, similar to our previous findings in SZ. We have now obtained identical measures in SZ and BDP where both patient samples have been chronically ill and on medication, but the pattern of findings has been different. Our own findings build on a large literature of WM abnormalities in SZ and BDP where studies with medication-naive individuals and with first-degree relatives have found changes consistent with those seen in chronic patients taking medication. Definitive investigation of medication effects on WM measures should include medication-naive patients; however, this is logistically difficult. In addition, our data were collected from a single voxel within the right prefrontal cortex, which limits generalization of study findings. Owing to the length of our current MRI scans, we were not able to study more than a single brain region. We selected the PFC as our focus in this study because it has been implicated in BD and WM abnormalities there may have impacts on emotional and cognitive processing, both of which are altered in BD. Extension of these methods to other WM regions will be informative.

Our finding of a role for myelin reductions in BDP is consistent with a large literature demonstrating abnormalities in oligodendrocyte cell counts, gene expression, and other abnormalities in the WM in BD (Drevets et al, 1998; Ongur et al, 1998; Tkachev et al, 2003). Future studies should focus on improving our understanding of oligoden- drocyte biology and therapeutic interventions that might reverse WM abnormalities in BDP.

\section{FUNDING AND DISCLOSURE}

This work was supported by the National Institutes of Health (KEL, Grant no. K23MH91210 and DO, Grant no. R01MH094594), Brain \& Behavior Research Foundation (FD, Grant no. 19299) and The Shervert Frazier Research Institute at the McLean Hospital (KEL). DO served on the scientific advisory board for Eli Lilly. KEL served as a consultant for Clintara. The authors declare no competing interests.

\section{REFERENCES}

Adler CM, Holland SK, Schmithorst V, Wilke M, Weiss KL, Pan H et al (2004). Abnormal frontal white matter tracts in bipolar disorder: a diffusion tensor imaging study. Bipolar Disord 6: 197-203.

Beyer JL, Taylor WD, MacFall JR, Kuchibhatla M, Payne ME, Provenzale JM et al (2005). Cortical white matter microstructural abnormalities in bipolar disorder. Neuropsychopharmacology 30: 2225-2229.

Beyer JL, Young R, Kuchibhatla M, Krishnan KR (2009). Hyperintense MRI lesions in bipolar disorder: a meta-analysis and review. Int Rev Psychiatry 21: 394-409.

Bruno SD, Barker GJ, Cercignani M, Symms M, Ron MA (2004). A study of bipolar disorder using magnetization transfer imaging and voxel-based morphometry. Brain 127: 2433-2440.

Burdick KE, Goldberg TE, Cornblatt BA, Keefe RS, Gopin CB, Derosse $\mathrm{P}$ et al (2011). The MATRICS consensus cognitive battery in patients with bipolar I disorder. Neuropsychopharmacology 36: 1587-1592.

Chen W, Luo Y, Merkle H, Zhu X-H, Adriany G, Garwood M et al (1995). Low-Power, B1-Insensitive, Frequency-Selective Saturation Pulse for use of Water Suppression and Saturation Transfer in NMR. SMR 3rd Annual Meeting: 1016.

Cui L, Chen Z, Deng W, Huang X, Li M, Ma X et al (2011). Assessment of white matter abnormalities in paranoid schizophrenia and bipolar mania patients. Psychiatry Res 194: 347-353. deGraff RA, Luo Y, Garwood M, Nicolay K (1996). B1-insensitive, single-shot localization and water suppression. J Magn Res Ser B 113: $35-45$.

Drevets WC, Ongur D, Price JL (1998). Neuroimaging abnormalities in the subgenual prefrontal cortex: implications for the pathophysiology of familial mood disorders. Mol Psychiatry 3: 220-226.

Du F, Cooper AJ, Thida T, Shinn AK, Cohen BM, Ongur D (2013). Myelin and axon abnormalities in schizophrenia measured with magnetic resonance imaging techniques. Biol Psychiatry 74: 451-457.

Du F, Ongur D (2013). Probing myelin and axon abnormalities separately in psychiatric disorders using MRI techniques. Front Integr Neurosci 7: 24.

Emsell L, Langan C, Van Hecke W, Barker GJ, Leemans A, Sunaert S et al (2013). White matter differences in euthymic bipolar I disorder: a combined magnetic resonance imaging and diffusion tensor imaging voxel-based study. Bipolar Disord 15: 365-376.

Kan HE, Techawiboonwong A, van Osch MJ, Versluis MJ, Deelchand DK, Henry PG et al (2012). Differences in apparent diffusion coefficients of brain metabolites between grey and white matter in the human brain measured at 7 T. Magn Reson Med 67: 1203-1209.

Kempton MJ, Geddes JR, Ettinger U, Williams SC, Grasby PM (2008). Meta-analysis, database, and meta-regression of 98 
structural imaging studies in bipolar disorder. Arch Gen Psychiatry 65: 1017-1032.

Laule C, Vavasour IM, Kolind SH, Li DK, Traboulsee TL, Moore GR et al (2007). Magnetic resonance imaging of myelin. Neurotherapeutics 4: 460-484.

Linke J, King AV, Poupon C, Hennerici MG, Gass A, Wessa M (2013). Impaired anatomical connectivity and related executive functions: differentiating vulnerability and disease marker in bipolar disorder. Biol Psychiatry 74: 908-916.

Liu J, Dietz K, DeLoyht JM, Pedre X, Kelkar D, Kaur J et al (2012). Impaired adult myelination in the prefrontal cortex of socially isolated mice. Nat Neurosci 15: 1621-1623.

Makinodan M, Rosen KM, Ito S, Corfas G (2012). A critical period for social experience-dependent oligodendrocyte maturation and myelination. Science 337: 1357-1360.

McDonald C, Bullmore ET, Sham PC, Chitnis X, Wickham H, Bramon E et al (2004). Association of genetic risks for schizophrenia and bipolar disorder with specific and generic brain structural endophenotypes. Arch Gen Psychiatry 61: 974-984.

McIntosh AM, Job DE, Moorhead TW, Harrison LK, Lawrie SM, Johnstone EC (2005). White matter density in patients with schizophrenia, bipolar disorder and their unaffected relatives. Biol Psychiatry 58: 254-257.

McIntosh AM, Munoz Maniega S, Lymer GK, McKirdy J, Hall J, Sussmann JE et al (2008). White matter tractography in bipolar disorder and schizophrenia. Biol Psychiatry 64: 1088-1092.

Mori S, Zhang J (2006). Principles of diffusion tensor imaging and its applications to basic neuroscience research. Neuron 51: 527-539.

Nortje G, Stein DJ, Radua J, Mataix-Cols D, Horn N (2013). Systematic review and voxel-based meta-analysis of diffusion tensor imaging studies in bipolar disorder. J Affect Disord 150: 192-200.

Nuechterlein KH, Green MF, Kern RS, Baade LE, Barch DM, Cohen JD et al (2008). The MATRICS consensus cognitive battery, part 1: test selection, reliability, and validity. Am J Psychiatry 165: 203-213.

Oertel-Knochel V, Reinke B, Alves G, Jurcoane A, Wenzler S, Prvulovic D et al (2014). Frontal white matter alterations are associated with executive cognitive function in euthymic bipolar patients. J Affect Disord 155: 223-233.
Ongur D, Drevets WC, Price JL (1998). Glial reduction in the subgenual prefrontal cortex in mood disorders. Proc Natl Acad Sci USA 95: 13290-13295.

Regenold WT, Phatak P, Marano CM, Gearhart L, Viens CH, Hisley KC (2007). Myelin staining of deep white matter in the dorsolateral prefrontal cortex in schizophrenia, bipolar disorder, and unipolar major depression. Psychiatry Res 151: 179-188.

Sarrazin S, Poupon C, Linke J, Wessa M, Phillips M, Delavest M et al (2014). A multicenter tractography study of deep white matter tracts in bipolar i disorder: psychotic features and interhemispheric disconnectivity. JAMA Psychiatry 71: 388-396.

Sokolov BP (2007). Oligodendroglial abnormalities in schizophrenia, mood disorders and substance abuse. Comorbidity, shared traits, or molecular phenocopies? Int J Neuropsychopharmacol 10: 547-555.

Stanfield AC, Moorhead TW, Job DE, McKirdy J, Sussmann JE, Hall J et al (2009). Structural abnormalities of ventrolateral and orbitofrontal cortex in patients with familial bipolar disorder. Bipolar Disord 11: 135-144.

Sussmann JE, Lymer GK, McKirdy J, Moorhead TW, Munoz Maniega S, Job D et al (2009). White matter abnormalities in bipolar disorder and schizophrenia detected using diffusion tensor magnetic resonance imaging. Bipolar Disord 11: 11-18.

Tkac I, Starcuk Z, Choi IY, Gruetter R (1999). In vivo 1H NMR spectroscopy of rat brain at $1 \mathrm{~ms}$ echo time. Magn Reson Med 41: 649-656.

Tkachev D, Mimmack ML, Ryan MM, Wayland M, Freeman T, Jones PB et al (2003). Oligodendrocyte dysfunction in schizophrenia and bipolar disorder. Lancet 362: 798-805.

Torgerson $\mathrm{CM}$, Irimia $\mathrm{A}$, Leow $\mathrm{AD}$, Bartzokis $\mathrm{G}$, Moody $\mathrm{TD}$, Jennings RG et al (2013). DTI tractography and white matter fiber tract characteristics in euthymic bipolar I patients and healthy control subjects. Brain Imaging Behav 7: 129-139.

Vederine FE, Wessa M, Leboyer M, Houenou J (2011). A metaanalysis of whole-brain diffusion tensor imaging studies in bipolar disorder. Prog Neuropsychopharmacol Biol Psychiatry 35: $1820-1826$.

Yurgelun-Todd DA, Silveri MM, Gruber SA, Rohan ML, Pimentel PJ (2007). White matter abnormalities observed in bipolar disorder: a diffusion tensor imaging study. Bipolar Disord 9: 504-512. 